\title{
HOCHSTER'S THETA PAIRING AND ALGEBRAIC EQUIVALENCE
}

\author{
OLGUR CELIKBAS AND MARK E. WALKER
}

\begin{abstract}
We define a variant of Hochster's $\theta$ pairing and prove that it is constant in flat families of modules over hypersurfaces with isolated singularities. As a consequence, we show that the $\theta$ pairing factors through the Grothendieck group modulo algebraic equivalence. Moreover, our result allows us, in certain situations, to translate the properties of the $\theta$ pairing in characteristic zero (established in [18 and [19) to the characteristic $p$ setting. We also give an application of our result to the rigidity of Tor over hypersurfaces.
\end{abstract}

\section{INTRODUCTION}

Let $R$ be the ring of regular functions of a hypersurface with isolated singularities - i.e., $R=S / f$ where $S$ is regular and the set

$$
\operatorname{Sing}(R)=\left\{p \in \operatorname{Spec}(R) \mid R_{p} \text { is not regular }\right\}
$$

is a finite set of maximal ideals. Then for any pair of finitely generated $R$-modules $M$ and $N$, for all $i \gg 0$, the Tor modules $\operatorname{Tor}_{i}^{R}(M, N)$ have finite length and are periodic of period at most two (i.e., $\operatorname{Tor}_{i}^{R}(M, N) \cong \operatorname{Tor}_{i+2}^{R}(M, N)$ ). Under these assumptions, Hochster's theta pairing 12 is defined as

$$
\theta^{R}(M, N)=\operatorname{length}\left(\operatorname{Tor}_{2 i}^{R}(M, N)\right)-\operatorname{length}\left(\operatorname{Tor}_{2 i+1}^{R}(M, N)\right) \text { for } i \gg 0 .
$$

The $\theta$ pairing is additive on short exact sequences in each argument, and thus determines a $\mathbb{Z}$-valued pairing on $G(R)$, the Grothendieck group of finitely generated $R$-modules. One looses no information by tensoring with $\mathbb{Q}$, and so often $\theta$ is interpreted as a symmetric bilinear form on the rational vector space $G(R)_{\mathbb{Q}}=G(R) \otimes \mathbb{Q}$.

Hochster [12] originally defined the theta pairing (for a larger class of pairs of modules) to study the direct summand conjecture. Recently the $\theta$ pairing was examined in detail in [6], 8] and [18. Dao [6, 2.8] observed an important connection of the $\theta$ pairing with the rigidity of Tor: Assume $(R, \mathfrak{m})=S /(f)$ where $S$ is an unramified (or equi-characteristic) regular local ring and $R_{\mathfrak{p}}$ is regular for all $\mathfrak{p} \neq \mathfrak{m}$. Then the vanishing of $\theta^{R}(M, N)$ implies that the pair of finitely generated $R$-modules $(M, N)$ is "Tor-rigid" in the sense that if $\operatorname{Tor}_{n}^{R}(M, N)=0$ for some non-negative integer $n$, then $\operatorname{Tor}_{i}^{R}(M, N)=0$ for all $i \geq n$. (We will record a slightly more general version of this fact in Proposition 3.4)

In [18, Moore et. al. study the $\theta$ pairing for rings of the form

$$
R=k\left[x_{0}, \ldots, x_{n}\right] / f\left(x_{0}, \ldots, x_{n}\right),
$$

where $k$ is an algebraically closed field and $f$ is a homogeneous polynomial of degree $d$ such that $X:=\operatorname{Proj}(R)$ is a smooth $k$-variety. (So, $\mathfrak{m}=\left(x_{0}, \ldots, x_{n}\right)$ is the only

2000 Mathematics Subject Classification. 13D03.

Key words and phrases. Rigidity of Tor, theta pairing, hypersurface singularities. 
non-smooth point of $R$.) They show that the $\theta$ pairing is induced, via the Chern character map, from the pairing on the primitive part of $\ell$-adic étale cohomology

given by

$$
\frac{H_{e t}^{\frac{n-1}{2}}\left(X, \mathbb{Q}_{\ell}\right)}{\mathbb{Q}_{\ell} \cdot \gamma^{\frac{n-1}{2}}} \times \frac{H_{e t}^{\frac{n-1}{2}}\left(X, \mathbb{Q}_{\ell}\right)}{\mathbb{Q}_{\ell} \cdot \gamma^{\frac{n-1}{2}}} \rightarrow \mathbb{Q}_{\ell}
$$

$$
(a, b) \mapsto\left(\int_{X} a \cup \gamma^{\frac{n-1}{2}}\right)\left(\int_{X} b \cup \gamma^{\frac{n-1}{2}}\right)-d \int_{X} a \cup b .
$$

(Here, $\ell$ is any prime distinct from $\operatorname{char}(k)$ and $\gamma$ is the class of a hyperplane section.) In particular, the theta pairing vanishes for rings $R$ of this type having even dimension (i.e., for which $n$ is even). Moreover, in the odd dimensional case, when $\operatorname{char}(k)=0$, using the Hodge-Riemann bilinear relations, they show that the above pairing on étale cohomology is positive/negative definite depending whether $n$ is congruent to 3 or $1(\bmod 4)$. In particular, $(-1)^{\frac{n+1}{2}} \theta$ is semi-positive definite in this case.

Closely related to the $\theta$ pairing is the Herbrand difference $h(M, N)$, introduced by Buchweitz [4, which is defined by replacing Tor with Ext in the formula defining $\theta$. Under mild hypotheses, these two invariants are essentially the same: If $R$ is finite type over a field, then upon tensoring with $\mathbb{Q}$, each pairing is induced from a pairing on the graded (rational) Chow group, $C H^{\cdot}(R)_{\mathbb{Q}}$, of $R$. On the summand $C H^{j}(R)_{\mathbb{Q}}$, the $\theta$ pairing and the Herbrand difference agree when $j$ is even, and they differ by a sign when $j$ is odd.

When $R$ has the form $\mathbb{C}\left[\left[x_{0}, \ldots, x_{n}\right]\right] / f$ with $f$ a power series with an isolated singularity, the Herbrand difference coincides with Euler characteristic for the category of matrix factorizations of $f$. Recently, Polishchuk and Vaintrob 19, Theorem 4.1.4] have established a "Riemann-Roch" formula of the form

$$
h(M, N)=\langle\operatorname{ch}(M), \operatorname{ch}(N)\rangle
$$

for maximum Cohen-Macaulay $R$-modules $M$ and $N$. Here, $\operatorname{ch}(M)$ is the "Chern character" of $M$, which is a certain element in the Milnor algebra of $f$ :

$$
\frac{\mathbb{C}\left[\left[x_{0}, \ldots, x_{n}\right]\right]}{\left(\frac{\partial f}{\partial x_{0}}, \ldots, \frac{\partial f}{\partial x_{n}}\right)} .
$$

The pairing $\langle-,-\rangle$ on the Milnor algebra is given by the formula involving generalized fractions

$$
\langle g, h\rangle=\operatorname{Res}\left[\frac{g h d x_{0} \wedge \cdots \wedge d x_{n}}{\frac{\partial f}{\partial x_{0}}, \ldots, \frac{\partial f}{\partial x_{n}}}\right] .
$$

The class $\operatorname{ch}(M)$ is given in terms of a matrix factorization of $f$ that presents $M$, and is defined in terms of the "boundary-bulk map", a construction arising in mathematical physics. In particular, $h(M, N)$ may be computed directly using the calculus of generalized fractions from the entries of the matrix factorization representations of $M$ and $N$. Moreover, since the boundary-bulk map vanishes when $n$ is even, this result shows that the $h$ pairing, and hence the $\theta$ pairing, vanish for even dimensional rings $R$ of this type. This exciting connection between homological algebra of hypersurfaces and mathematical physics is only beginning to be explored.

The main point of this article is to establish the invariance of the $\theta$ pairing in flat families - see Theorem 2.7 for a precise statement. For example, suppose $A$ is 
a complete dvr, $f$ belongs to $C:=A\left[x_{0}, \ldots, x_{n}\right]$, and $B:=C / f$ is flat over $A$ and its singular locus is module finite over $A$. Suppose also that $M$ and $N$ are a pair of finitely generated $B$-modules that are $A$-flat. Then given a map $A \rightarrow L$ with $L$ an algebraically closed field, the integer $\theta^{B \otimes_{A} L}\left(M \otimes_{A} L, N \otimes_{A} L\right)$ is independent of $L$ (and the map $A \rightarrow L$ ).

In particular, taking $A$ to be the $p$-adic integers, our result allows us to translate, in certain situations, results in characteristic 0 , such as those listed above, to results in characteristic $p$ - see Corollary 4.5 for precise statements of this sort. In the equi-characteristic setting, if we take $A$ to be the ring of regular functions of a smooth affine curve over an algebraically closed field $k$, then our results imply that the $\theta$ pairing does not distinguish between algebraically equivalent elements of $G(R)$. In other words, $\theta$ descends to a pairing on the Grothendieck group of $R$ modulo algebraic equivalence — see Corollary 3.3 for a precise statement.

\section{Main Theorem}

Throughout this paper, all rings are assumed to be commutative and Noetherian. We give some definitions used in our main theorem.

Definition 2.1. Let $R$ be a ring. An $R$-algebra $S$ is essentially of finite type over $R$ if $S$ is a localization of a finitely generated $R$-algebra. $S$ is said to be essentially smooth over $R$ if (i) $S$ is essentially of finite type over $R$, (ii) the map $R \rightarrow S$ is flat, and (iii) for all ring maps $R \rightarrow L$ where $L$ is a field, the ring $L \otimes_{R} S$ is regular.

For example, if $k$ is a field and $S$ is a smooth $k$-algebra - that is,

$$
S=k\left[x_{1}, \ldots, x_{n}\right] /\left(f_{1}, \ldots, f_{m}\right)
$$

and the Jacobian matrix $\left(\frac{\partial f_{i}}{\partial x_{j}}\right) \otimes_{S} S / \mathfrak{m}$ has rank equal to $n-\operatorname{dim}\left(S_{\mathfrak{m}}\right)$ for each maximal ideal $\mathfrak{m}$ of $S$ - then any localization of $S$ is essentially smooth over $k$.

Definition 2.2. $A$ flat family of hypersurfaces with isolated singularities consists of a base ring $A$, an $A$-algebra $C$, and an element $f \in C$ such that, letting $B:=C /(f)$, the following conditions hold:

(1) $\operatorname{Spec}(A)$ is connected,

(2) $C$ is essentially smooth over $A$,

(3) $B$ is flat over $A$, and

(4) there exists an ideal $I$ of $B$ such that $B / I$ is module finite over $A$ and $B_{\mathfrak{p}}$ is essentially smooth over $A$ for all $\mathfrak{p} \in \operatorname{Spec}(B) \backslash V_{B}(I)$.

Remark 2.3. For the ideal $I$ in Definition 2.2 $V(I)$ contains the singular locus of the map $A \rightarrow B$, and so the assumption that $A \rightarrow B / I$ is module finite implies that $A \rightarrow B$ is a family of isolated singularities. In more detail, given $A \rightarrow L$ with $L$ a field, for $q \in \operatorname{Spec}\left(B \otimes_{A} L\right)$, let $p=q \cap B$. Then $\left(B \otimes_{A} L\right)_{q}$ is a localization of $\left(B \otimes_{A} L\right)_{p}$ and $\left(B \otimes_{A} L\right)_{p} \cong B_{p} \otimes_{A} L$. In particular, if $p$ is a regular point of $\operatorname{Spec}(B)$, then $q$ is a regular point of $\operatorname{Spec}\left(B \otimes_{A} L\right)$. It follows that $\operatorname{Sing}\left(B \otimes_{A} L\right) \subseteq V_{B \otimes_{A} L}(J)$, where $J:=I \cdot\left(B \otimes_{A} L\right)$. Since $\left(B \otimes_{A} L\right) / J \cong(B / I) \otimes_{A}$ $L$ is a finite dimensional $L$-algebra, $\operatorname{Sing}\left(B \otimes_{A} L\right)$ is a finite set of maximal ideals. Also notice that $B \otimes_{A} L=\left(C \otimes_{A} L\right) /(f)$ and $C \otimes_{A} L$ is regular, by assumption, and hence $B \otimes_{A} L$ is a hypersurface with isolated singularities. 
Example 2.4. Suppose $A$ is a complete dvr (e.g., the $p$-adic integers) and $C=$ $A\left[x_{0}, \ldots, x_{n}\right]_{\left(x_{0}, \ldots, x_{n}\right)}$. Say $f \in C$ is such that $\left(\frac{\partial f}{\partial x_{0}}, \cdots, \frac{\partial f}{\partial x_{n}}\right)$ (where $\frac{\partial-}{\partial x_{i}}$ is the evident $A$-linear derivation of $C$ ) contains $\left(x_{0}^{N}, \ldots, x_{n}^{N}\right)$ for some $N \gg 0$. For example, $f=x_{0}^{d_{0}}+\cdots+x_{n}^{d_{n}}$ satisfies this condition provided the integers $d_{0}, \ldots, d_{n}$ are invertible in $A$. Then $A, C$ and $f \in C$ form a flat family of hypersurfaces with isolated singularities. The required ideal $I$ may be taken to be $\left(\frac{\partial f}{\partial x_{0}}, \cdots, \frac{\partial f}{\partial x_{n}}\right)$.

Definition 2.5. For a flat family of hypersurfaces with isolated singularities as in Definition 2.2. if $M$ and $N$ are finitely generated $B$-modules that are $A$-flat and $\alpha: A \rightarrow L$ is a ring map with $L$ a field, we define

$$
\begin{aligned}
\tilde{\theta}_{\alpha}(M, N):=\operatorname{dim}_{L} & \left(\operatorname{Tor}_{2 i}^{B \otimes_{A} L}\left(M \otimes_{A} L, N \otimes_{A} L\right)\right)- \\
& \operatorname{dim}_{L}\left(\operatorname{Tor}_{2 i-1}^{B \otimes_{A} L}\left(M \otimes_{A} L, N \otimes_{A} L\right)\right) \text { for } i \gg 0 .
\end{aligned}
$$

Since $B \otimes_{A} L$ is a hypersurface, we have

$$
\operatorname{Tor}_{i}^{B \otimes_{A} L}\left(M \otimes_{A} L, N \otimes_{A} L\right) \cong \operatorname{Tor}_{i+2}^{B \otimes_{A} L}\left(M \otimes_{A} L, N \otimes_{A} L\right)
$$

for all $i \gg 0$. Moreover, since $\operatorname{Sing}\left(B \otimes_{A} L\right) \subset V_{B \otimes_{A} L}\left(I \cdot\left(B \otimes_{A} L\right)\right)$ and $\left(B \otimes_{A} L\right) / I$. $\left(B \otimes_{A} L\right)$ is finite dimensional over $L$ by Remark 2.3] it follows that $\operatorname{Tor}_{i}^{B \otimes_{A} L}\left(M \otimes_{A} L, N \otimes_{A} L\right)$ is supported on $V\left(I \cdot\left(B \otimes_{A} L\right)\right)$ and is finite dimensional over $L$, for all $i \gg 0$. In particular, $\widetilde{\theta}_{\alpha}(M, N)$ is well-defined.

Remark 2.6. The pairing $\widetilde{\theta}_{\alpha}$ is closely related to the $\theta$ pairing. For observe that if $L$ is algebraically closed, then the residue of every prime in the Artinian ring $\left(B \otimes_{A}\right.$ $L) / I \cdot\left(B \otimes_{A} L\right)$ is isomorphic to $L$ (by Nullstellensatz). Since $\operatorname{Tor}_{i}^{B} \otimes_{A} L\left(M \otimes_{A} L, N \otimes_{A} L\right)$ is supported on $V\left(I \cdot\left(B \otimes_{A} L\right)\right)$ for all $i \gg 0$, it follows that

$$
\operatorname{dim}_{L} \operatorname{Tor}_{i}^{B \otimes_{A} L}\left(M \otimes_{A} L, N \otimes_{A} L\right)=\operatorname{length}_{B \otimes_{A} L} \operatorname{Tor}_{i}^{B \otimes_{A} L}\left(M \otimes_{A} L, N \otimes_{A} L\right)
$$

for all $i \gg 0$, and hence

$$
\widetilde{\theta}_{\alpha}(M, N)=\theta^{B \otimes_{A} L}\left(M \otimes_{A} L, N \otimes_{A} L\right)
$$

for such L. Typically, for questions involving $\theta$, passage along a faithfully flat extension looses no information, and thus one may reduce to a situation in which the residue fields are algebraically closed.

The main result of this paper is the following. (See Definitions 2.2 and 2.5 for the terminology.)

Theorem 2.7. Let $A, C$, and $f \in C$ be a flat family of hypersurfaces with isolated singularities. Set $B=C / f$ and let $M$ and $N$ be finitely generated $B$-modules both of which are flat as $A$-modules. If $\alpha: A \rightarrow L$ is a ring map with $L$ a field, then $\widetilde{\theta}_{\alpha}(M, N)$ is independent of $L$ and $\alpha$.

Example 2.8. Let $A=\mathbb{Z}, C=\mathbb{Z}\left[x_{1}, \ldots, x_{n}, y_{1}, \ldots, y_{n}\right], f=\sum_{i=1}^{n} x_{i} y_{i}, M=B /\left(x_{1}, \ldots, x_{n}\right)$ and $N=B /\left(y_{1}, \ldots, y_{n}\right)$. Then $A, C, f$ form a flat family of hypersurfaces with isolated singularities (for which $I$ may be taken to be $\left(x_{0}, \ldots, x_{n}, y_{0}, \ldots, y_{n}\right)$ ). Our Theorem 2.7 implies $\widetilde{\theta}_{\alpha}(M, N)$ is independent of $\alpha$. This can be checked directly: $\operatorname{Tor}_{i}^{B \otimes_{A} L}\left(M \otimes_{A} L, N \otimes_{A} L\right) \cong L$ if $i \geq 0$ is even and $\operatorname{Tor}_{i}^{B \otimes_{A} L}\left(M \otimes_{A} L, N \otimes_{A} L\right)=0$ if $i$ is odd. (See, for example, [5, 3.12].) Therefore $\widetilde{\theta}_{\alpha}(M, N)=1$ for all $\alpha$. 
Proof of Theorem 2.7. Let $E$ be a field and let $\beta: A \rightarrow E$ be a ring map; it suffices to prove that $\widetilde{\theta}_{\alpha}(M, N)=\widetilde{\theta}_{\beta}(M, N)$. Note that, for some $p \in \operatorname{Spec}(A)$, we have a commutative diagram of ring maps of the form:

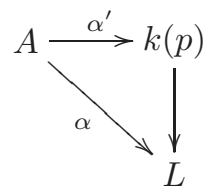

Moreover

$$
\operatorname{Tor}_{i}^{B \otimes_{A} L^{\prime}}\left(M \otimes_{A} L^{\prime}, N \otimes_{A} L^{\prime}\right) \otimes_{L^{\prime}} L \cong \operatorname{Tor}_{i}^{B \otimes_{A} L}\left(M \otimes_{A} L, N \otimes_{A} L\right)
$$

where $L^{\prime}=k(p)$, and hence $\widetilde{\theta}_{\alpha}(M, N)=\widetilde{\theta}_{\alpha^{\prime}}(M, N)$. Without loss of generality we may therefore assume $L=k(p)$ and $E=k(q)$, for some $p, q \in \operatorname{Spec}(A)$. Since $\operatorname{Spec}(A)$ is connected, we may also assume that $p \subseteq q$ and $\operatorname{ht}(q)=\operatorname{ht}(p)+1$.

Set $A^{\prime}=\widetilde{A}_{\mathfrak{n}}$ where $\widetilde{A}$ is the integral closure of $A_{q} / p A_{q}$ in its field of fractions and $\widetilde{\mathfrak{n}}$ is a maximal ideal of $\widetilde{A}$. Then we have a commutative diagram of ring maps of the form:

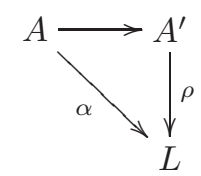

Moreover, the isomorphism

$$
\begin{aligned}
\operatorname{Tor}_{i}^{\left(B \otimes_{A} A^{\prime}\right) \otimes_{A^{\prime}} L} & \left(\left(M \otimes_{A} A^{\prime}\right) \otimes_{A^{\prime}} L,\left(N \otimes_{A} A^{\prime}\right) \otimes_{A^{\prime}} L\right) \\
& \cong \operatorname{Tor}_{i}^{B \otimes_{A} L}\left(M \otimes_{A} L, N \otimes_{A} L\right)
\end{aligned}
$$

shows that $\widetilde{\theta}_{\alpha}(M, N)=\widetilde{\theta}_{\rho}\left(M \otimes_{A} A^{\prime}, N \otimes_{A} A^{\prime}\right)$. Since the properties of being flat and essentially smooth are preserved under base change, $A^{\prime}, C \otimes_{A} A^{\prime}, B \otimes_{A} A^{\prime}$, $M \otimes_{A} A^{\prime}$ and $N \otimes_{A} A^{\prime}$ satisfy the hypotheses of the Theorem. Without loss of generality we may therefore assume $A$ is a dvr with unique maximal ideal $\mathfrak{n}, L$ is the field of fractions of $A$ and $E$ is the residue field $A / \mathfrak{n}$.

Set $\mathfrak{n}=(\pi)$ and fix a sufficiently large integer $j$. We claim

$$
\operatorname{Supp}_{B}\left(\operatorname{Tor}_{j}^{B}(M, N)\right) \subseteq V_{B}(I) .
$$

Indeed, if $q^{\prime} \notin V_{B}(I)$, then $B_{q^{\prime}}$ is essentially smooth over $A$. If $A \rightarrow B_{q^{\prime}}$ is a local map, then by [16, 23.7] $B_{q^{\prime}}$ is regular. Otherwise, $\pi$ is a unit in $B_{q^{\prime}}$ and hence

$$
B_{q^{\prime}} \otimes_{A} A\left[\frac{1}{\pi}\right]=B_{q^{\prime}}\left[\frac{1}{\pi}\right]=B_{q^{\prime}}
$$

is regular. Either way, $\operatorname{Tor}_{j}^{B}(M, N)_{q^{\prime}}=0$.

It follows that $I^{l} \cdot \operatorname{Tor}_{j}^{B}(M, N)=0$ for some $l>0$. Using the fact that $B / I$ is a finitely generated $A$-module, we see that $\operatorname{Tor}_{n}^{B}(M, N)$ is a finitely generated $A$-module for all $n \gg 0$. Therefore, for all $n \gg 0$, we can write

$$
\operatorname{Tor}_{n}^{B}(M, N)=A^{\left(r_{n}\right)} \oplus G_{n}
$$


where $r_{n}$ is some integer and $G_{n}$ is the torsion part of $\operatorname{Tor}_{n}^{B}(M, N)$. Let $t_{n}=\operatorname{dim}_{E}\left(\frac{G_{n}}{\pi G_{n}}\right)$. Since $N$ is $A$-flat, we have a short exact sequence of complexes

$$
0 \rightarrow P_{\bullet} \otimes_{B} N \stackrel{\pi}{\rightarrow} P \bullet \otimes_{B} N \rightarrow\left(P \bullet \otimes_{B} N\right) \otimes_{A} E \rightarrow 0 .
$$

where $P_{\bullet} \stackrel{\sim}{\longrightarrow} M$ is a free resolution of $M$ over $B$. Since $B$ is flat over $A, P_{\bullet}$ is also a flat $A$-resolution of $M$. Furthermore, as $M$ is $A$-flat, $\mathrm{H}_{n}\left(P_{\bullet} \otimes_{A} E\right)=0$ for all $n \geq 1$. Therefore $P_{\bullet} \otimes_{A} L \stackrel{\sim}{\longrightarrow} M \otimes_{A} L$ is a free resolution of $M \otimes_{A} L$ over $B \otimes_{A} L$, and hence

$$
\operatorname{Tor}_{n}^{B \otimes_{A} L}\left(M \otimes_{A} L, N \otimes_{A} L\right) \cong \mathrm{H}_{n}\left(\left(P \bullet \otimes_{B} N\right) \otimes_{A} L\right) .
$$

Similarly,

$$
\operatorname{Tor}_{n}^{B \otimes_{A} E}\left(M \otimes_{A} E, N \otimes_{A} E\right) \cong \mathrm{H}_{n}\left(\left(P \bullet \otimes_{B} N\right) \otimes_{A} E\right) .
$$

Now (2.2) and (2.4) yield the exact sequence

$$
\operatorname{Tor}_{i}^{B}(M, N) \stackrel{\pi}{\rightarrow} \operatorname{Tor}_{i}^{B}(M, N) \rightarrow \operatorname{Tor}_{i}^{B \otimes_{A} E}\left(M \otimes_{A} E, N \otimes_{A} E\right) \rightarrow \operatorname{Tor}_{i-1}^{B}(M, N) .
$$

Notice (2.3) implies $\operatorname{Tor}_{n}^{B \otimes_{A} L}\left(M \otimes_{A} L, N \otimes_{A} L\right) \cong \operatorname{Tor}_{n}^{B}(M, N) \otimes_{A} L$ since $L$ is $A$ flat. Thus, by (2.1), $\operatorname{Tor}_{j}^{B \otimes_{A} L}\left(M \otimes_{A} L, N \otimes_{A} L\right) \cong L^{\left(r_{j}\right)}$ and hence $\widetilde{\theta}_{\alpha}(M, N)=r_{2 j}-r_{2 j-1}$. We have, by (2.5), the short exact sequences

$$
0 \rightarrow C_{i} \rightarrow \operatorname{Tor}_{i}^{B \otimes_{A} E}\left(M \otimes_{A} E, N \otimes_{A} E\right) \rightarrow K_{i-1} \rightarrow 0
$$

where $K_{i}$ and $C_{i}$ are the kernel and cokernel of the multiplication by $\pi$ on $\operatorname{Tor}_{i}^{R}(M, N)$, respectively. Now it follows from (2.1) and (2.6) that

$$
\tilde{\theta}_{\beta}(M, N)=\left(r_{2 j}+t_{2 j}+t_{2 j-1}\right)-\left(r_{2 j-1}+t_{2 j-1}+t_{2 j-2}\right) .
$$

Since $\operatorname{Tor}_{n}^{B}(M, N) \cong \operatorname{Tor}_{n+2}^{B}(M, N)$ for all $n \gg 0$, we conclude that

$$
\widetilde{\theta}_{\alpha}(M, N)=r_{2 j}-r_{2 j-1}=\widetilde{\theta}_{\beta}(M, N) \text {. }
$$

\section{Some Consequences of the Main Theorem}

We explain how the main theorem implies that the $\theta$ pairing factors through "algebraic equivalence". For this, it is helpful to record the following simplified version of the theorem.

Corollary 3.1. Assume $k$ is a field, $S$ is essentially smooth over $k, f$ is an element of $S$ such that the singular locus, $\operatorname{Sing}(R)$, of $R:=S / f$ is a finite set of maximal ideals, and that $R / \mathfrak{m} \cong k$ for every $\mathfrak{m} \in \operatorname{Sing}(R)$ in the singular locus. (The latter condition holds, for example, if $k$ is algebraically closed and $R$ is finitely generated as a $k$-algebra). If $A$ is a Noetherian $k$-algebra such that $\operatorname{Spec}(A)$ is connected and $M$ and $N$ are finitely generated $R \otimes_{k} A$-modules that are flat as $A$-modules, then

$$
\theta^{R \otimes_{k} k(q)}\left(M \otimes_{A} k(q), N \otimes_{A} k(q)\right)
$$

is independent of $q \in \operatorname{Spec}(A)$. 
Proof. This follows by applying the theorem to the flat family of hypersurfaces with isolated singularities given by $A, C=S \otimes_{k} A$, and $f \otimes 1 \in C$. The fact that $R / \mathfrak{m} \cong k$ for all $\mathfrak{m} \in \operatorname{Sing}(R)$ implies that

$$
\widetilde{\theta}_{\alpha}(M, N)=\theta^{R \otimes_{k} L}\left(M \otimes_{A} L, N \otimes_{A} L\right)
$$

where $\alpha: A \rightarrow L$ is any ring map with $L$ a field.

Definition 3.2. For a finitely generated $k$-algebra $R$ with $k$ algebraically closed, we define algebraic equivalence on $G(R)$ as the equivalence relation $\sim$ generated by the following elementary relation: Given classes $\alpha$ and $\beta$ in $G(R), \alpha \sim \beta$ if there exist a finitely generated, smooth $k$-algebra $A$ with $\operatorname{Spec}(A)$ connected, maximal ideals $\mathfrak{m}_{1}$ and $\mathfrak{m}_{2}$ of $A$ and a class $\gamma \in G\left(R \otimes_{k} A\right)$ such that $\alpha=i_{1}(\gamma)$ and $\beta=i_{2}(\gamma)$, where

$$
i_{\epsilon}: G\left(R \otimes_{k} A\right) \rightarrow G(R)
$$

is the homomorphism defined on generators by

$$
[M] \mapsto \sum_{j \geq 0}(-1)^{j}\left[\operatorname{Tor}_{j}^{A}\left(M, A / \mathfrak{m}_{\epsilon}\right)\right] .
$$

Observe that $\operatorname{Tor}_{j}^{A}\left(M, A / \mathfrak{m}_{\epsilon}\right)$ is an $R \otimes_{k} A / \mathfrak{m}_{\epsilon}$-module and, since $k \cong A / \mathfrak{m}_{\epsilon}$, we have that $R \cong R \otimes_{k} A / \mathfrak{m}_{\epsilon}$.

Corollary 3.3. If $k$ an algebraically closed field, $S$ is a smooth $k$-algebra, and $R=S / f$ is a hypersurface with isolated singularities, then the $\theta$ pairing on $G(R)$ factors through algebraic equivalence; that is, there is a commutative diagram of the form:

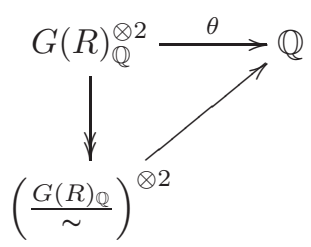

Proof. For a smooth $k$-algebra $A$ such that $\operatorname{Spec}(A)$ is connected, a pair of maximal ideals $\mathfrak{m}_{1}, \mathfrak{m}_{2}$ of $A$, and a finitely generated $R \otimes_{k} A$-module $M$ that is $A$-flat, we apply Corollary 3.1 to $M$ and $N=T \otimes_{k} A$, where $T$ is a finitely generated $R$-module. This gives $\theta\left(M_{1}, T\right)=\theta\left(M_{2}, T\right)$, where $M_{\epsilon}:=M \otimes_{A} A / \mathfrak{m}_{\epsilon}$. Since $G\left(R \otimes_{k} A\right)$ is generated by classes of modules that are flat over $A$, it follows that $\theta(-, T)$ annihilates the image of $i_{1}-i_{2}: G\left(R \otimes_{k} A\right) \rightarrow G(R)$.

Proposition 3.4. (H. Dao, cf. [6, 2.8]) Let $R=S /(f)$ and let $M$ and $N$ be finitely generated $R$-modules. Assume $\operatorname{Sing}(R)=\left\{\overline{\mathfrak{n}}_{1}, \overline{\mathfrak{n}}_{2}, \ldots, \overline{\mathfrak{n}}_{r}\right\}$ where $\mathfrak{n}_{1}, \ldots, \mathfrak{n}_{r}$ are maximal ideals of $S$ such that $S_{\mathfrak{n}_{i}}$ is an unramified regular local ring for each $i$. If $\theta^{R}(M, N)=0$, then $(M, N)$ is Tor-rigid over $R$.

Proof. Suppose that $\operatorname{Tor}_{n}^{R}(M, N)=0$ for some $n \geq 0$. Since rigidity holds over regular local rings ([1, Corollary 2.2] and [15, Corollary 1]), $\operatorname{Supp}\left(\operatorname{Tor}_{i}^{R}(M, N)\right) \subseteq$ $\operatorname{Sing}(R)$ for all $i \geq n$. Hence length $\left(\operatorname{Tor}_{i}^{R}(M, N)\right)<\infty$ for all $i \geq n$. As $\theta^{R}(M, N)=$ $\theta^{T^{-1} R}\left(T^{-1} M, T^{-1} N\right)$ for $T=R-\bigcup_{i} \overline{\mathfrak{n}}_{i}$, we may assume $S$ is semi-local with maximal ideals $\mathfrak{n}_{1}, \mathfrak{n}_{2}, \ldots, \mathfrak{n}_{r}$. Then

$$
\chi_{n}^{S}(M, N)=\chi_{n}^{S_{\mathfrak{n}_{1}}}\left(M_{\mathfrak{n}_{1}}, N_{\mathfrak{n}_{1}}\right)+\cdots+\chi_{n}^{S_{\mathfrak{n}_{r}}}\left(M_{\mathfrak{n}_{r}}, N_{\mathfrak{n}_{r}}\right)
$$


where $\chi_{n}^{S}(M, N)=\sum_{j \geq n}(-1)^{j-n} \cdot \operatorname{length}\left(\operatorname{Tor}_{j}^{R}(M, N)\right)$ is the higher Euler characteristic of the pair $(M, N)$. Write

$$
\theta^{R}(M, N)=\operatorname{length}\left(\operatorname{Tor}_{2 e}^{R}(M, N)\right)-\operatorname{length}\left(\operatorname{Tor}_{2 e-1}^{R}(M, N)\right)
$$

for some $e \gg 0$ and consider the following exact sequence [20]:

$$
\begin{aligned}
0 \rightarrow \operatorname{Tor}_{2 e}^{R}(M, N) \rightarrow & \cdots \rightarrow \operatorname{Tor}_{n}^{R}(M, N) \\
& \rightarrow \operatorname{Tor}_{n+1}^{S}(M, N) \rightarrow \operatorname{Tor}_{n+1}^{R}(M, N) \rightarrow C \rightarrow 0
\end{aligned}
$$

Taking the alternating sum of the lengths, we deduce that:

$$
\operatorname{length}(C)+\chi_{n+1}^{S}(M, N)=(-1)^{2 e-n} \cdot \theta_{R}(M, N)+\operatorname{length}\left(\operatorname{Tor}_{n}^{R}(M, N)\right)
$$

It follows from [13] and [15] that, for all $t \geq n, \chi_{t}^{S_{\mathfrak{n}_{i}}}\left(M_{\mathfrak{n}_{i}}, N_{\mathfrak{n}_{i}}\right) \geq 0$ and $\chi_{t}^{S_{\mathfrak{n}_{i}}}\left(M_{\mathfrak{n}_{i}}, N_{\mathfrak{n}_{i}}\right)=0$

if and only if $\operatorname{Tor}_{j}^{S_{\mathfrak{n}_{i}}}\left(M_{\mathfrak{n}_{i}}, N_{\mathfrak{n}_{i}}\right)=0$ for all $j \geq t$. Thus $C=0$ and $\chi_{n+1}^{S}(M, N)=0$.

This implies $\operatorname{Tor}_{n+1}^{R}(M, N)=0$ and hence $\operatorname{Tor}_{i}^{R}(M, N)=0$ for all $i \geq n$.

Using Dao's result (as extended in Proposition 3.4), we may use Theorem 2.7 to give a statement about Tor-rigidity:

Corollary 3.5. Assume $A, C, f, B=C / f$ form a flat family of hypersurfaces with isolated singularities and $M, N$ are finitely generated $B$-modules that are $A$-flat. Let $\alpha: A \rightarrow L$ and $\beta: A \rightarrow E$ be ring maps where $L$ and $E$ are fields. If $\widetilde{\theta}_{\alpha}(M, N)=0$, then the pair $\left(M \otimes_{A} E, N \otimes_{A} E\right)$ is Tor-rigid over $B \otimes_{A} E$. In particular, if $\operatorname{pd}_{B \otimes_{A} L}\left(M \otimes_{A} L\right)<\infty$, then the pair $\left(M \otimes_{A} E, N \otimes_{A} E\right)$ is Tor-rigid over $B \otimes_{A} E$.

Proof. It follows from Theorem 2.7 that $\widetilde{\theta}_{\gamma}(M, N)=0$, where $\gamma$ is the ring map from $A$ to the algebraic closure $\bar{E}$ of $E$ induced by $\beta$. In view of Remark 2.6 we have $\theta^{B \otimes_{A} \bar{E}}\left(M \otimes_{A} \bar{E}, N \otimes_{A} \bar{E}\right)=0$. Hence, by Proposition 3.4, the pair $\left(M \otimes_{A}\right.$ $\left.\bar{E}, N \otimes_{A} \bar{E}\right)$ is Tor-rigid over $B \otimes_{A} \bar{E}$. The result now follows from the fact that $B \otimes_{A} E \longrightarrow B \otimes_{A} \bar{E}$ is faithfully flat. The final assertion holds since the $\theta$ pairing clearly vanishes if either argument has finite projective dimension.

It is worth mentioning that $\operatorname{pd}_{B \otimes_{A} L}\left(M \otimes_{A} L\right)<\infty$ does not imply $\operatorname{pd}_{B \otimes_{A} E}\left(M \otimes_{A}\right.$ $E)<\infty$ in general:

Example 3.6. Let $k$ be a field with $\operatorname{char}(k) \neq 2$ and let $A=k[t], C=k[x, y, t]$, $L=k(t), E=A /(t), f=y^{2}-x(x-t)$ and $M=B /(x, y)$. Then $B \otimes_{A} L=$ $k(t)[x, y] /(f)$ is regular since it is smooth over $k(t)$. Thus $\operatorname{pd}_{B \otimes_{A} L}\left(M \otimes_{A} L\right)<\infty$. However, as $B \otimes_{A} E \cong k[x, y] /\left(y^{2}-x^{2}\right)$ is singular at $(x, y), M \otimes_{A} E \cong k$ is of infinite projective dimension over $B \otimes_{A} E$.

\section{Applications to Matrix Factorizations}

In certain situations it is possible to lift a given hypersurface with isolated singularities to a non-trivial flat family of such. For example, starting with one in characteristic $p$, it is sometimes possible to lift it to a family that is indexed by a mixed characteristic ring. In some situations, this allows one to deduce results in the characteristic $p$ setting from known results in the characteristic 0 setting. 
Proposition 4.1. Let $F$ be a field, $S=F\left[x_{1}, \ldots, x_{n}\right]$ and $f \in\left(x_{1}, \ldots, x_{n}\right) \subset S$. Assume $\left(x_{1}, \ldots, x_{n}\right)$ is an isolated point in the locus of non-smooth points of $S / f$ - i.e., assume there exists $g \in S \backslash\left(x_{1}, \ldots, x_{n}\right)$ such that $\operatorname{Spec}\left((S / f)\left[\frac{1}{g}\right]\right)$ $\left\{\left(x_{1}, \ldots, x_{n}\right)\right\}$ is smooth over $F$. Let $\left(A, \mathfrak{m}_{A}\right)$ be a Henselian local ring (e.g., a complete local ring) with residue field $F$ and let $\widetilde{S}=A\left[x_{1}, \ldots, x_{n}\right]$ and $\mathfrak{n}=\mathfrak{m}_{A}+$ $\left(x_{1}, \ldots, x_{n}\right)$.

For any $\widetilde{f} \in \widetilde{S}$ such that $\widetilde{f} \equiv f\left(\bmod \mathfrak{m}_{A}\right)$, there exits $h \in \widetilde{S} \backslash \mathfrak{n}$ such that $(\widetilde{S} / \widetilde{J})\left[\frac{1}{h}\right]$ is a finitely generated $A$-module, where $\widetilde{J}=\left(\frac{\partial \widetilde{f}}{\partial x_{1}}, \ldots, \frac{\partial \widetilde{f}}{\partial x_{n}}, \widetilde{f}\right)$ and $\frac{\partial-}{\partial x_{i}}$ are the evident A-linear derivations.

Proof. We may assume $\widetilde{J} \subseteq \mathfrak{n}$, for otherwise we may pick $h$ to be any element of $\widetilde{J}-\mathfrak{n}$.

Let $T=\widetilde{S} / \widetilde{J}$. Since $A \rightarrow T$ has finite type, by the upper semi-continuity of fiber dimensions 10, 13.1.3], the set of primes $q \in \operatorname{Spec}(T)$ such that $\operatorname{dim}\left((T /(q \cap A))_{q}=\right.$ 0 is an open subset of $\operatorname{Spec}(T)$. Since $(T /(\mathfrak{n} \cap A))_{\mathfrak{n}} \cong(S / f)_{\left(x_{1}, \ldots, x_{n}\right)}$ and $\left(x_{1}, \ldots, x_{n}\right)$ is an isolated singularity of $S / f$, we get that $\mathfrak{n}$ belongs to this open subset. It follows that there exists an $\alpha \in T \backslash \mathfrak{n}$ such that $A \rightarrow V:=T\left[\frac{1}{\alpha}\right]$ is quasi-finite.

Since $A$ is a Henselian local ring, it follows from [17, I.4.2] that $V=V_{0} \times V_{1} \times \cdots \times$ $V_{m}$ where $V_{i}$ is a finitely generated $A$-module for all $i=1, \ldots, m$ and $\mathfrak{p} \cap A=\{0\}$ for all $\mathfrak{p} \in \operatorname{Spec}\left(V_{0}\right)$. Identifying $\operatorname{Spec}(V)$ with the disjoint union of $\operatorname{Spec}\left(V_{i}\right)$, we see that $\mathfrak{n} \notin \operatorname{Spec}\left(V_{0}\right)$. Without loss of generality, assume $\mathfrak{n} \in \operatorname{Spec}\left(V_{1}\right)$, so that $\left(V_{i}\right)_{\mathfrak{n}}=0$ for $i \neq 1$. Then there exits $\beta \in V \backslash \mathfrak{n}$ such that $V_{1}\left[\frac{1}{\beta}\right]=V_{1}$ and $V_{i}\left[\frac{1}{\beta}\right]=0$ for $i \neq 1$.

Now set $h=\alpha \cdot \beta$. Then $(\widetilde{S} / \widetilde{J})\left[\frac{1}{h}\right] \cong V\left[\frac{1}{\beta}\right] \cong V_{1}$ is a finitely generated $A$ module.

In the homogeneous case, the previous result may be refined:

Proposition 4.2. Let $F$ be a field, $S=F\left[x_{1}, \ldots, x_{n}\right]$ and $f \in S$ be a homogeneous element such that $\left(x_{1}, \ldots, x_{n}\right)$ is the only non-smooth point of $S / f$. Let $A$ be a complete local ring with residue field $F$. Let $C=A\left[x_{1}, \ldots, x_{n}\right]$ and let $\tilde{f} \in C$ be any homogeneous element such that $\tilde{f} \equiv f\left(\bmod \mathfrak{m}_{A}\right)$. Set $B=C /(\widetilde{f})$ and $I=\left(\frac{\partial \widetilde{f}}{\partial x_{1}}, \ldots, \frac{\partial \widetilde{f}}{\partial x_{n}}\right) \subset B$. Then $B / I$ is a finitely generated $A$-module.

Proof. Observe that $B / I$ is a graded $C$-module. By Proposition 4.1 there exists an $h \notin \mathfrak{m}_{A}+\left(x_{1}, \ldots, x_{n}\right)$ such that $(B / I)\left[\frac{1}{h}\right]$ is a finitely generated $A$-module. Consider a filtration

$$
0=M_{0} \subseteq M_{1} \subseteq \cdots \subseteq M_{n}=B / I
$$

by graded submodules, such that $M_{i} / M_{i-1} \cong C / q_{i}$ where each $q_{i}$ is a homogeneous prime ideal. Then $q_{i} \subseteq \mathfrak{m}_{A}+\left(x_{1}, \ldots, x_{n}\right)$ and hence $h \notin q_{i}$ for each $i$. Since $M_{i} / M_{i-1} \hookrightarrow\left(M_{i} / M_{i-1}\right)\left[\frac{1}{h}\right]$ is injective and $(B / I)\left[\frac{1}{h}\right]$ is module finite over $A$, it follows that the modules $M_{i} / M_{i-1}$, and hence $B / I$, are finitely generated $A$ modules.

Definition 4.3. (9]) For a ring $V$ and homogeneous element $f \in V\left[x_{0}, \ldots, x_{n}\right]$ (equipped with the standard grading), a homogeneous matrix factorization of $f$ is pair of $m \times m$ matrices $(A, B)$ with entries in $V\left[x_{0}, \ldots, x_{n}\right]$ such that each determines a map of graded free modules and $A B=f I_{m}=B A$. 
Remark 4.4. The condition that a $p \times q$ matrix $A=\left(a_{i, j}\right)$ with entries in $S:=$ $V\left[x_{0}, \ldots, x_{n}\right]$ determines a map of graded free modules is equivalent to the condition that every entry of $A$ is homogeneous and there are integers $\left(e_{1}, \ldots, e_{q}\right),\left(d_{1}, \ldots d_{p}\right)$ so that $\left|a_{i, j}\right|=e_{j}-d_{i}$ for all $i, j$. For given such integers, the map

$$
A: \bigoplus_{j=1}^{q} S\left(-e_{j}\right) \rightarrow \bigoplus_{i=1}^{p} S\left(-d_{i}\right)
$$

is a map of graded free modules, i.e., homogeneous of degree 0.

For a homogeneous matrix factorization $(A, B)$ of $f \in S:=F\left[x_{0}, \ldots, x_{n}\right]$ with $F$ a field, the cokernel of $A$ (viewed as a map of graded free modules) is a graded maximal Cohen-Macaulay module (cf. [3]) over $R:=F\left[x_{0}, \ldots, x_{n}\right] /(f)$. Conversely, if $M$ is a graded maximal Cohen-Macaulay $R$-module, then picking a graded free resolution of it as an $S$-module leads to a homogeneous matrix factorization.

As mentioned in the introduction, the theta pairing is known to vanish for hypersurfaces with isolated singularities of even dimension that are of the form $R=$ $F\left[x_{0}, \ldots, x_{n}\right](f)$ with $f$ homogeneous and $F$ any field $\left[18\right.$ or $R=\mathbb{C}\left[\left[x_{0}, \ldots, x_{n}\right]\right] /(g)$ 19. Moreover, in the homogeneous case with $n=\operatorname{dim}(R)$ odd and $\operatorname{char}(F)=0$, the pairing $(-1)^{\frac{n+1}{2}} \theta$ is semi-positive definite. The following result indicates a method of translating this fact to the positive characteristic setting in favorable situations.

Corollary 4.5. Let $F$ be an arbitrary field and let $S=F\left[x_{0}, \ldots, x_{n}\right]$ be given the standard grading where $n$ is odd. Let $(A, B)$ be a homogeneous matrix factorization of a homogeneous element $f \in S$ such that $\left(x_{0}, \ldots, x_{n}\right)$ is the only non-smooth prime of $R=S / f$.

Suppose there exist a complete dvr $\left(V, \mathfrak{m}_{V}\right)$ with residue field $F$ and field of fractions of characteristic 0 , a homogeneous element $\tilde{f} \in V\left[x_{0}, \ldots, x_{n}\right]$ and a homogeneous matrix factorization $(\tilde{A}, \tilde{B})$ of $\tilde{f}$ such that $\tilde{f} \equiv f, \tilde{A} \equiv A$ and $\tilde{B} \equiv B$ modulo $\mathfrak{m}_{V}$. Then $\theta(M, M) \geq 0$ if $n \equiv 3(\bmod 4)$ and $\theta(M, M) \leq 0$ if $n \equiv 1(\bmod 4)$.

Proof. Let $\tilde{M}$ be the graded $V\left[x_{0}, \ldots, x_{n}\right]$-module given by the cokernel of $\tilde{A}$. Then by Proposition 4.2 $V, V\left[x_{0}, \ldots, x_{n}\right]$, and $\tilde{f}$ from a flat family of hypersurfaces with isolated singularities. Moreover, $\tilde{M}$ is a flat $V$-module and hence by Theorem 2.7 we have $\tilde{\theta}_{\alpha}(\tilde{M}, \tilde{M})=\tilde{\theta}_{\beta}(\tilde{M}, \tilde{M})$ where $\alpha: V \rightarrow F$ is the map to the residue field of $B$ and $\beta: V \rightarrow L$ is the map to its field of fractions. Since the only singular prime of $R$, respectively $R^{\prime}:=L\left[x_{0}, \ldots, x_{n}\right] / \tilde{f}$, has residue field $F$, respectively $L$, we have $\tilde{\theta}_{\alpha}(\tilde{M}, \tilde{M})=\theta^{R}(M, N)$ and $\tilde{\theta}_{\beta}(\tilde{M}, \tilde{M})=\theta^{R^{\prime}}(M, N)$. The result now follows from [18, Theorem 3.4].

Example 4.6. Let $F$ be a field of characteristic $p>0$ and let $f \in S=F\left[x_{0}, \ldots, x_{n}\right]$ be a homogeneous element such that $\left(x_{0}, \ldots, x_{n}\right)$ is the only non-smooth prime of $S / f$. If $(A, B)$ is a homogeneous matrix factorization for $f$ satisfying the additional constraint that $\operatorname{det}(A)=f$, then a lifting $(\tilde{A}, \tilde{B})$ as in Corollary 4.5 always exists. Namely, pick any complete dvr of mixed characteristic $V$ with residue field $F$ (e.g., the ring of Witt vectors [2]) and let $\widetilde{A}$ be any matrix with coefficients in $V\left[x_{0}, \ldots, x_{n}\right]$ such that $\widetilde{A} \equiv A\left(\bmod \mathfrak{m}_{V}\right)$. Then set $\widetilde{B}=\widetilde{A}^{\text {adj }}$, and we get $\widetilde{A} \cdot \widetilde{B}=\widetilde{f} \cdot I_{r}$ with $\tilde{f} \equiv f\left(\bmod \mathfrak{m}_{V}\right)$. Thus, for such matrix factorizations, we have $\theta(M, M) \geq 0$ if $n \equiv 3(\bmod 4)$, and $\theta(M, M) \leq 0$ if $n \equiv 1(\bmod 4)$, where $M=\operatorname{coker}(A)$. 
Assume $R$ is a $d$-dimensional local hypersurface that is an isolated singularity. If $d \geq 4$, then there are no non-free maximal Cohen-Macaulay $R$-modules of rank one. (We are grateful to Hailong Dao and Roger Wiegand for explaining this to us.) For by a theorem of Grothendieck [11, if $R_{p}$ is factorial for all $p \in \operatorname{Spec}(R)$ such that height $(p) \leq 3$, then $R$ is factorial. Since a maximal Cohen-Macaulay module $M$ of rank one determines an element in the divisor class group of $R, M$ must be free.

On the other hand, rank one maximal Cohen-Macaulay modules over three dimensional hypersurface singularities exist. For example, the ideal $(x, y)$ of $R=$ $k[[x, y, z, w]] /(x w-y z)$ is a non-free rank one maximal Cohen-Macaulay module. In case $d=3$, Dao and the second author of this paper proved that $\theta^{R}(M, M) \geq 0$ for any finitely generated $R$-module $M$. (See also [7.)

The condition $\operatorname{det}(A)=f$ is equivalent to the condition that $M$ is a rank one maximal Cohen-Macaulay module (cf., for example, [14, 2.2.5]). Therefore the conclusion in Example 4.6 also follows from the discussion above.

\section{REFERENCES}

[1] M. Auslander. Modules over unramified regular local rings. Illinois J. Math., 5:631-647, 1961.

[2] N. Bourbaki. Éléments de mathématique. Algèbre commutative. Chapitres 8 et 9. Springer, Berlin, 2006. Reprint of the 1983 original.

[3] W. Bruns and J. Herzog. Cohen-Macaulay rings, volume 39 of Cambridge Studies in Advanced Mathematics. Cambridge University Press, Cambridge, 1993.

[4] R.-O. Buchweitz. Maximal Cohen-Macaulay modules and Tate cohomology over Gorenstein rings. preprint, (1986).

[5] O. Celikbas and H. Dao. Asymptotic behavior of Ext functors for modules of finite complete intersection dimension. in press. Math. Z., 2010. Posted at arXiv:1004.0294.

[6] H. Dao. Decency and Tor-rigidity for modules over hypersurfaces. preprint, arxiv:0611568, 2008.

[7] H. Dao. Picard groups of punctured spectra of dimension three local hypersurfaces are torsionfree. preprint, Arxiv: 1004.0471, 2010.

[8] H. Dao. Some observations on local and projective hypersurfaces. Math. Res. Lett., 15(2):207$219,2008$.

[9] D. Eisenbud. Homological algebra on a complete intersection, with an application to group representations. Trans. Amer. Math. Soc., 260(1):35-64, 1980.

[10] A. Grothendieck. Éléments de géométrie algébrique. IV. Étude locale des schémas et des morphismes de schémas. III. Inst. Hautes Études Sci. Publ. Math., (28):255, 1966.

[11] Alexander Grothendieck. Cohomologie locale des faisceaux cohérents et théorèmes de Lefschetz locaux et globaux (SGA 2). North-Holland Publishing Co., Amsterdam, 1968. Augmenté d'un exposé par Michèle Raynaud, Séminaire de Géométrie Algébrique du Bois-Marie, 1962, Advanced Studies in Pure Mathematics, Vol. 2.

[12] Melvin Hochster. The dimension of an intersection in an ambient hypersurface. In Algebraic geometry (Chicago, Ill., 1980), volume 862 of Lecture Notes in Math., pages 93-106. Springer, Berlin, 1981.

[13] Melvin Hochster. Euler characteristics over unramified regular local rings. Illinois. J. Math., 28:281-288, 1984.

[14] B. Hovinen. Matrix factorizations of the classical discriminant. Ph.D. Thesis, University of Toronto, 2009.

[15] S. Lichtenbaum. On the vanishing of Tor in regular local rings. Illinois J. Math., 10:220-226, 1966.

[16] H. Matsumura. Commutative ring theory, volume 8 of Cambridge Studies in Advanced Mathematics. Cambridge University Press, Cambridge, second edition, 1989. Translated from the Japanese by M. Reid.

[17] James S. Milne. Étale cohomology, volume 33 of Princeton Mathematical Series. Princeton University Press, Princeton, N.J., 1980. 
[18] W. F. Moore, G. Piepmeyer, S. Spiroff, and M.E. Walker. Hochster's theta invariant and the Hodge-Riemann bilinear relations. in press. Adv. Math. (2010). Posted at arXiv:0910.1289.

[19] A. Polishchuk and A. Vaintrob. Chern characters and Hirzebruch-Riemann-Roch formula for matrix factorizations. Preprint, arXiv:1002.

[20] J. Rotman. An introduction to homological algebra. Universitext. Springer, New York, second edition, 2009 .

Department of Mathematics, University of Kansas, 645 Snow Hall, 1460 Jayhawk Blvd, Lawrence, KS 66045-7523, USA

E-mail address: ocelikbas@math.ku.edu

Department of Mathematics, University of Nebraska-Lincoln, 328 Avery Hall, LinCOLN, NE 68588-0323, USA

E-mail address: mwalker@math.unl.edu 